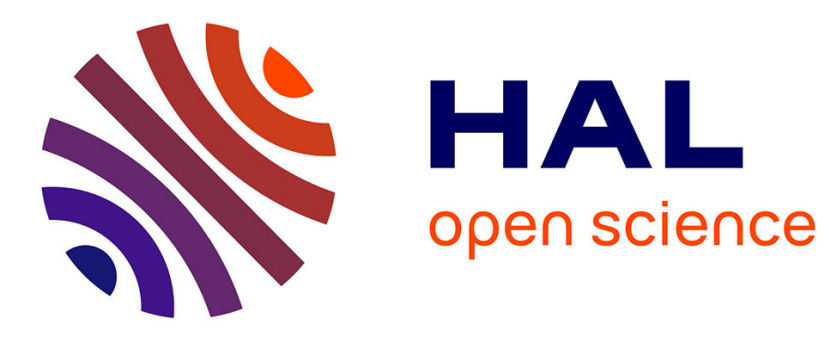

\title{
Homogenization of transport problems and semigroups
}

Etienne Bernard, François Golse, Francesco Salvarani

\section{To cite this version:}

Etienne Bernard, François Golse, Francesco Salvarani. Homogenization of transport problems and semigroups. Mathematical Methods in the Applied Sciences, 2010, 33 (10), pp.1228-1234. 10.1002/mma.1319 . hal-00472237

\section{HAL Id: hal-00472237 \\ https://hal-polytechnique.archives-ouvertes.fr/hal-00472237}

Submitted on 10 Apr 2010

HAL is a multi-disciplinary open access archive for the deposit and dissemination of scientific research documents, whether they are published or not. The documents may come from teaching and research institutions in France or abroad, or from public or private research centers.
L'archive ouverte pluridisciplinaire HAL, est destinée au dépôt et à la diffusion de documents scientifiques de niveau recherche, publiés ou non, émanant des établissements d'enseignement et de recherche français ou étrangers, des laboratoires publics ou privés. 


\title{
HOMOGENIZATION OF TRANSPORT PROBLEMS AND SEMIGROUPS
}

\author{
ETIENNE BERNARD, FRANÇOIS GOLSE, AND FRANCESCO SALVARANI
}

\begin{abstract}
In some cases, the homogenization of evolution differential equations whose solution is given by the action of a semigroup on their initial data may lead to evolution problems with a completely different structure, usually integro-differential equations whose dynamics is not defined by a semigroup, but involves memory effects. A typical example is the homogenization - or discretization — of opacities in radiative transfer. In this paper, we propose a formulation of homogenized equations in terms of a semigroup acting on an enlarged phase space (i.e. on functions involving more variables than in the original problem.)
\end{abstract}

To the memory of Aldo Belleni-Morante (1938-2009)

\section{INTRODUCTION}

The mathematical modeling of the response of composite materials to external fields usually involves partial differential equations with oscillating coefficients. Specifically, the wavelengths of these oscillations correspond with the spatial scales defined by the microscopic structure of the composite, i.e. the scale at which the elementary constituents of the composite are assembled. When investigating the macroscopic properties of such a composite material, a first step is to average out the oscillations of the coefficients at microscopic scale, and to filter the high frequency oscillations they induce in the response fields one is interested in. This mathematical process is called homogenization, since it may be viewed as the replacement of a composite material by an equivalent homogeneous material. In the most favorable cases, this would be done by simply replacing the response coefficients oscillating at microscopic scale in the field equation with coefficients for the equivalent homogeneous material where the oscillations at microscopic scale have been eliminated.

Unfortunately, this picture is outrageously optimistic. In many cases, a single response coefficient oscillating at microscopic scale will be replaced with several homogenized equivalent coefficients, for instance due to the persistence of anisotropy effects in the microscopic structure of the composite material. Worse, the structure of the partial differential equation itself can be modified after taking the homogenization limit, and this is precisely our concern in the present work. A striking example of such a change in the structure of the homogenized equation was given by Tartar [10], who observed that the homogenization limit of the simplest imaginable ordinary

2000 Mathematics Subject Classification. 35B27 (45J05, 85A25).

Key words and phrases. Homogenization, Integro-differential equations, Radiative transfer, Opacities. 
differential equation would lead to an integro-differential equation (i.e. involving memory terms). In other words, the group property of the original evolution equation can be destroyed by the homogenization limit.

Some time later, Vanderhaegen [11, 12], Levermore-Pomraning-SanzoWong [5], and Sentis [8] studied in detail the homogenization problem for the absorption coefficient in transport theory (for either neutrons or photons). Their work also leads to integro-differential equations as in the simple example considered by Tartar - and for the same basic reason.

The phenomena observed by Tartar in his simple example - i.e. the fact that the group property satisfied by the solutions of an evolution equation can be destroyed by the homogenization limit - also occurs in very different contexts. It has been very recently identified in a classical problem in nonequilibrium statistical mechanics, namely the Boltzmann-Grad limit of the periodic Lorentz gas, by E. Caglioti and the second author, and by J. Marklof and Strömbergsson in [3, 2, 6], as well as in a homogenization problem for the linear Boltzmann equation in a periodically perforated domain, by the two first authors and E. Caglioti [1]. In all these works, the solution of the equation at microscopic scale is given by a semigroup, and, in order to keep the semigroup property after passing to the macroscopic limit, it is necessary to consider an enlarged phase space involving additional variables. The present paper explains how the ideas in [2, 6, 1] can be applied in the context of the homogenization of opacities considered in [11, 12, 5, 7, 8].

Semigroups and kinetic models have been among Aldo Belleni-Morante's favorite scientific subjects. His own ideas have had a great influence on the development of this field of mathematical analysis. In view of his own particular interest in photonics and, more generally, transport problems in astrophysics, we dedicate this modest contribution to his memory.

\section{Homogenization of An ODE}

Our starting point is the following elementary, yet fairly instructive example, due to L. Tartar 10].

Let $a \in L^{\infty}\left(\mathbf{T}^{N}\right)$, assume without loss of generality that $a \geq 0$ a.e. on $\mathbf{T}^{N}$, and consider, for each $\epsilon>0$, the ODE with unknown $u_{\epsilon} \equiv u_{\epsilon}(t, z) \in \mathbf{R}$ :

$$
\left\{\begin{array}{l}
\frac{d u_{\epsilon}}{d t}+a\left(\frac{z}{\epsilon}\right) u_{\epsilon}=0, \quad t>0, z \in \mathbf{R}^{N}, \\
u_{\epsilon}(0, z)=u^{i n}(z)
\end{array}\right.
$$

where $u^{i n} \in L^{2}\left(\mathbf{R}^{N}\right) \cap L^{\infty}\left(\mathbf{R}^{N}\right)$. Obviously, for each $\epsilon>0$, one has

$$
u_{\epsilon}(t, z)=u^{i n}(z) e^{-t a(z / \epsilon)}, \quad t>0, z \in \mathbf{R}^{N},
$$

so that, in the limit as $\epsilon \rightarrow 0^{+}$, one has

$$
u_{\epsilon} \rightarrow u \text { in } L^{\infty}\left(\mathbf{R}_{+} \times \mathbf{R}^{N}\right) \text { weak-* }
$$

where the limit $u$ is explicitly given by the formula

$$
u(t, z)=u^{i n}(z) \Phi(t), \quad t \geq 0, z \in \mathbf{R}^{N},
$$

with

$$
\Phi(t)=\int_{\mathbf{T}^{N}} e^{-t a(y)} d y, \quad t \geq 0 .
$$


This example shows that the homogenized solution $u$ does not satisfy the equation

$$
\frac{d u}{d t}+\bar{a} u=0
$$

where $\bar{a}$ is the average of $a$ on $\mathbf{T}^{N}$, i.e.

$$
\bar{a}=\int_{\mathbf{T}^{N}} a(y) d y,
$$

as someone unfamiliar with the intricacies of homogenization might (naively) expect. Worse, unless $a$ is a.e. constant on $\mathbf{T}^{N}$, there does not exist any $A \in \mathbf{R}$ such that

$$
\frac{d u}{d t}+A u=0 .
$$

(Should such an $A$ exist, it would be referred to as the "homogenized coefficient" equivalent to the oscillating coefficient $a(z / \epsilon)$.) Equivalently, although for each $\epsilon>0$ the solution $u_{\epsilon}$ is defined in terms of $u^{i n}$ by the semigroup $S_{\epsilon}(t)$ defined on $L^{2}\left(\mathbf{R}^{N}\right)$ by the formula

$$
S_{\epsilon}(t) \phi=\phi(z) e^{-t a(z / \epsilon)},
$$

the homogenized solution $u$ is not given in terms of $u^{i n}$ by a semigroup acting on $L^{2}\left(\mathbf{R}^{N}\right)$, since (by convexity of the exponential)

$$
\Phi\left(t_{1}+t_{2}\right) \neq \Phi\left(t_{1}\right) \Phi\left(t_{2}\right), \quad t_{1}, t_{2}>0,
$$

unless $a$ is a.e. constant on $\mathbf{T}^{N}$ - meaning that there are no fast oscillations in the original problem (2.1), so that there is no need for homogenization in this case.

In fact, L. Tartar (see lecture 35 in [10]) proved that the homogenized solution satisfies the following integro-differential equation

$$
\left\{\begin{array}{l}
\frac{d u}{d t}(t, z)+\bar{a} u(t, z)=\int_{0}^{t} K(t-s) u(s, z) d s, \quad t>0, z \in \mathbf{R}^{N}, \\
u(0, z)=u^{i n}(z),
\end{array}\right.
$$

where the Laplace transform of $K$ is given by the expression

$\tilde{K}(p):=\int_{0}^{\infty} e^{-p t} K(t) d t=\int_{\mathbf{T}^{N}}(p+a(y)) d y-\left(\int_{\mathbf{T}^{N}} \frac{d y}{p+a(y)} d y\right)^{-1}, \quad p>0$.

Concerning the appearance of an integro-differential equation such as (2.5) as the homogenization limit of an ODE, it is instructive to compare the situation above with the problem

$$
\left\{\begin{array}{l}
\frac{d v_{\epsilon}}{d t}+b\left(\frac{t}{\epsilon}\right) v_{\epsilon}=0, \quad t>0 \\
v_{\epsilon}(0)=v^{i n}
\end{array}\right.
$$

with unknown $v_{\epsilon} \equiv v_{\epsilon}(t) \in \mathbf{R}$, where $b \in L^{\infty}\left(\mathbf{T}^{1}\right)$. In this case

$$
v_{\epsilon}(t)=v^{i n} \exp \left(-\int_{0}^{t} b(s / \epsilon) d s\right) \rightarrow v^{i n} e^{-B t}=v(t)
$$


for each $t \geq 0$ as $\epsilon \rightarrow 0^{+}$, where

$$
B:=\int_{0}^{1} b(\sigma) d \sigma .
$$

Indeed,

$$
\frac{1}{t} \int_{0}^{t} b(s / \epsilon) d s=\frac{\epsilon}{t} \int_{0}^{t / \epsilon} b(\sigma) d \sigma \rightarrow \lim _{t \rightarrow+\infty} \frac{1}{t} \int_{0}^{t} b(\sigma) d \sigma=B
$$

as $\epsilon \rightarrow 0^{+}$. Hence, the homogenized equation obtained from (2.6) is

$$
\left\{\begin{array}{l}
\frac{d v}{d t}+B v=0, \quad t>0, \\
v(0)=v^{i n},
\end{array}\right.
$$

and in this case, $B$ is the equivalent absorption coefficient obtained from the oscillating absorption coefficient $b(t / \epsilon)$ by homogenization.

The difference between the homogenization of problems (2.1) and (2.6) is that in the latter case, the oscillating variable in the coefficient $b$ is the time variable, and the equation (2.6) provides a bound on the time derivative of the solution $v_{\epsilon}$, thereby excluding the possibility of fast oscillations in $t$ in the solution $v_{\epsilon}$.

At variance with this case, in Tartar's example (2.1), the oscillating variable is $z$, and the equation (2.1) does not involve derivatives in $z$ to prevent the buildup of fast oscillations in $z$ in the solution $u_{\epsilon}$. In that example, the fast oscillations in $z$ in both $a(z / \epsilon)$ and $u_{\epsilon}$ combine to produce the integral term on the right-hand side of (2.5).

Obviously, the example (2.1) can be generalized to the case where the quasi-periodic oscillating coefficient $a(z / \epsilon)$ is replaced with a bounded family $a_{\epsilon} \equiv a_{\epsilon}(z)$ of functions in $L^{\infty}\left(\mathbf{R}^{N}\right)$ converging in the sense of Young measures as $\epsilon \rightarrow 0^{+}$.

\section{A SEMigroup In EXTENDED PHASE SPACE}

As a warm-up, we shall in this section consider again Tartar's example above, and express the homogenization limit of (2.1) in terms of a semigroup defined on an extended phase space - i.e. acting on functions with additional variables.

Let $a_{\epsilon} \equiv a_{\epsilon}(z)$ be a bounded family of functions in $L^{\infty}\left(\mathbf{R}^{N}\right)$ converging in the sense of Young measures to $\left(\mu_{z}\right)_{z \in \mathbf{R}^{N}}$ (see [9] for a lucid presentation of the notion of Young measures.) In other words, $\left(\mu_{z}\right)_{z \in \mathbf{R}^{N}}$ is a family of probability measures on $\mathbf{R}$ that measurably depends on $z$, and satisfies, for each $f \in C_{b}(\mathbf{R})$

$$
f\left(a_{\epsilon}\right) \rightarrow F_{a} \text { in } L^{\infty}\left(\mathbf{R}^{N}\right) \text { weak-* }{ }^{*} \text { with } F_{a}(z)=\int_{\mathbf{R}} f(\lambda) d \mu_{z}(\lambda)=:\left\langle\mu_{z}, f\right\rangle
$$

in the limit as $\epsilon \rightarrow 0^{+}$. Without loss of generality, we henceforth assume that $a_{\epsilon} \geq \alpha>0$ a.e. on $\mathbf{R}^{N}$.

For each $\epsilon>0$, let $u_{\epsilon} \equiv u_{\epsilon}(t, z)$ be the solution of

$$
\left\{\begin{array}{l}
\frac{d u_{\epsilon}}{d t}+a_{\epsilon}(z) u_{\epsilon}=0, \quad t>0, z \in \mathbf{R}^{N}, \\
u_{\epsilon}(0, z)=u^{i n}(z)
\end{array}\right.
$$


where $u^{i n} \in L^{1} \cap L^{\infty}\left(\mathbf{R}^{N}\right)$.

Proposition 3.1. In the limit as $\epsilon \rightarrow 0^{+}$, one has

$$
u_{\epsilon} \rightarrow u=\int_{0}^{+\infty} U d s \text { in } L^{\infty}\left(\mathbf{R}_{+} \times \mathbf{R}^{N}\right) \text { weak- }{ }^{*},
$$

where $U \equiv U(t, s, z)$ is the solution of

$$
\left\{\begin{array}{l}
\partial_{t} U-\partial_{s} U=0, \quad t, s>0, z \in \mathbf{R}^{N}, \\
U(0, s, z)=-u^{i n}(z) \frac{d \tilde{\mu}_{z}}{d s}(s) .
\end{array}\right.
$$

(We recall that the notation $\tilde{\mu}_{z}$ designates the Laplace transform of $\mu_{z}$.)

Before giving the (elementary) proof of this result, a few remarks are in order.

First, the equation satisfied by $U$ is a free transport equation, where $s \in \mathbf{R}_{+}$is the space variable. Since the vector field $-\partial_{s}$ is outgoing on the boundary of the half-line $\mathbf{R}_{+}$, there is no need of a boundary condition for $s=0$, so that the problem (3.2) is well-posed - in $L^{2}\left(\mathbf{R}_{+} \times \mathbf{R}^{N} ; e^{-s} d s d z\right)$, for instance.

Next, although the homogenization limit $u$ of $u_{\epsilon}$ as $\epsilon \rightarrow 0^{+}$is not of the form $u(t, \cdot)=S(t) u^{i n}$ with $S(t)$ a semigroup on $L^{2}\left(\mathbf{R}^{N}\right)$, the function $U$ is defined by a semigroup in terms of its initial data (since the equation satisfied by $U$ is a free transport equation.) Specifically

$U(t, s, z)=\Sigma(t) U(0, s, z)$ with $\Sigma(t) \psi(s, z)=\psi(t+s, z), \quad t, s>0, z \in \mathbf{R}^{N}$.

In other words, while there does not exist any semigroup $S(t)$ acting on $L^{2}\left(\mathbf{R}^{N}\right)$ such that $S_{\epsilon}(t) \rightarrow S(t)$ in the weak operator topology for each $t>0$ as $\epsilon \rightarrow 0^{+}$, one has

$$
S_{\epsilon}(t) \rightarrow \int_{0}^{+\infty} \Sigma(t) d s
$$

in that same topology.

Proof. For each $\epsilon>0$, define

$$
U_{\epsilon}(t, s, z):=u_{\epsilon}(t, z) a_{\epsilon}(z) e^{-s a_{\epsilon}(z)}, \quad t, s \geq 0, z \in \mathbf{R}^{N} .
$$

Obviously

$$
\left(\partial_{t}-\partial_{s}\right) U_{\epsilon}(t, s, z)=a_{\epsilon}(z) e^{-s a_{\epsilon}(z)}\left(\frac{d u_{\epsilon}}{d t}(t, z)+a_{\epsilon}(z) u_{\epsilon}(t, z)\right)
$$

so that $U_{\epsilon}$ satisfies

$$
\left\{\begin{array}{l}
\partial_{t} U_{\epsilon}-\partial_{s} U_{\epsilon}=0, \quad t, s>0, z \in \mathbf{R}^{N}, \\
U_{\epsilon}(0, s, z)=u^{i n}(z) a_{\epsilon}(z) e^{-s a_{\epsilon}(z)}
\end{array}\right.
$$

Since $a_{\epsilon}>0$ a.e. on $\mathbf{R}^{N}$, one has $\left\|U_{\epsilon}(t, \cdot, \cdot)\right\|_{L^{\infty}\left(\mathbf{R}_{+} \times \mathbf{R}^{N}\right)} \leq\left\|u^{i n}\right\|_{L^{\infty}\left(\mathbf{R}^{N}\right)}$. Hence $U_{\epsilon}$ is bounded and therefore (by the Banach-Alaoglu theorem) relatively weak-* compact in $\left.L^{\infty}\left(\mathbf{R}_{+} \times \mathbf{R}_{+} \times \mathbf{R}^{N}\right)\right)$. If $U$ is a weak-* limit point of $U_{\epsilon}$ as $\epsilon \rightarrow 0^{+}$, by passing to the limit in the sense of distributions in the free transport equation satisfied by $U_{\epsilon}$, we conclude that $\left(\partial_{t}-\partial_{s}\right) U=0$. 
Since $U_{\epsilon}$ is bounded in $L^{\infty}\left(\mathbf{R}_{+} \times \mathbf{R}_{+} \times \mathbf{R}^{N}\right)$, the free transport equation satisfied by $U_{\epsilon}$ implies that $\partial_{t} U_{\epsilon}$ is bounded in $L^{\infty}\left(\mathbf{R}_{+} \times \mathbf{R}^{N} ; W^{-1, \infty}\left(\mathbf{R}_{+}\right)\right)$. Therefore, $\left.\left.U_{\epsilon_{n}}\right|_{t=0} \rightarrow U\right|_{t=0}$ in $\left.L^{\infty}\left(\mathbf{R}^{N} ; W^{-1, \infty}\left(\mathbf{R}_{+}\right)\right)\right)$weak-* for each subsequence $\epsilon_{n} \downarrow 0$ such that $U_{\epsilon_{n}} \rightarrow U$ in $L^{\infty}\left(\mathbf{R}_{+} \times \mathbf{R}_{+} \times \mathbf{R}^{N}\right)$ weak-*. Since

$$
\begin{array}{r}
\left.U_{\epsilon}\right|_{t=0} \rightarrow u^{i n}(z) \int_{0}^{+\infty} a e^{-s a} d \mu_{z}(a)=-u^{i n}(z) \frac{d \tilde{\mu}_{z}}{d s}(s) \\
\text { in } L^{\infty}\left(\mathbf{R}_{+} \times \mathbf{R}^{N}\right) \text { weak-* }
\end{array}
$$

and the problem (3.2) has a unique solution, $U_{\epsilon} \rightarrow U$ in $L^{\infty}\left(\mathbf{R}_{+} \times \mathbf{R}_{+} \times \mathbf{R}^{N}\right)$ weak-* $^{*}$ as $\epsilon \rightarrow 0^{+}$.

Since $a_{\epsilon}(z) \geq \alpha>0$ a.e. in $z \in \mathbf{R}^{N}$, one has

$$
\int_{T}^{+\infty}\left|U_{\epsilon}(t, s, z)\right| d s=e^{-T a_{\epsilon}(z)}\left|u_{\epsilon}(t, z)\right| \leq e^{-T \alpha}\left\|u^{i n}\right\|_{L^{\infty}\left(\mathbf{R}^{N}\right)}
$$

so that, for each test function $\phi \in L^{1}\left(\mathbf{R}_{+} \times \mathbf{R}^{N}\right)$,

$$
\int_{0}^{+\infty} \int_{\mathbf{R}^{N}}\left(\int_{T}^{+\infty}\left|U_{\epsilon}(t, s, z)\right| d s\right)|\phi(t, z)| d t d z \rightarrow 0 \text { uniformly in } \epsilon>0
$$

as $T \rightarrow+\infty$, by dominated convergence. Since on the other hand

we conclude that

$$
u_{\epsilon}(t, z)=\int_{0}^{+\infty} U_{\epsilon}(t, s, z) d s
$$

$$
u_{\epsilon}=\int_{0}^{+\infty} U_{\epsilon} d s \rightarrow \int_{0}^{+\infty} U d s
$$

in $L^{\infty}\left(\mathbf{R}_{+} \times \mathbf{R}^{N}\right)$ weak-*

\section{Homogenization of opacities in RADiative transfer}

In this section, we shall apply the method described above to the equation of radiative transfer.

Radiative transfer is a kinetic theory for a gas of photons exchanging energy with a background material (such as a plasma, a stellar or a planetary atmosphere). This energy exchange is the result of absorption, emission or scattering of photons by the atoms in the background matter. The state at time $t$ of the population of photons is given by the specific radiative intensity denoted $I(t, x, \omega, \nu)$ that is $c h \nu$ times the number density of photons with frequency $\nu$ located at the position $x$ with direction $\omega$. Here, $c$ is the speed of light while $h$ is Planck's constant.

Neglecting scattering processes, the radiative intensity satisfies the radiative transfer equation

$$
\frac{1}{c} \partial_{t} I+\omega \cdot \nabla_{x} I=\sigma(\nu, T) B_{\nu}(T)-\sigma(\nu, T) I .
$$

Here $B_{\nu}(T)$ is the specific radiative intensity at frequency $\nu$ of a black body at temperature $T$, while $\sigma(\nu, T)>0$ is the opacity, or absorption crosssection per unit volume, of the background material at temperature $T$ for an incident radiation with frequency $\nu$. While $B_{\nu}(T)$ has the explicit expression

$$
B_{\nu}(T)=\frac{2 h \nu^{3}}{c^{2}} \frac{1}{e^{h \nu / k T}-1},
$$




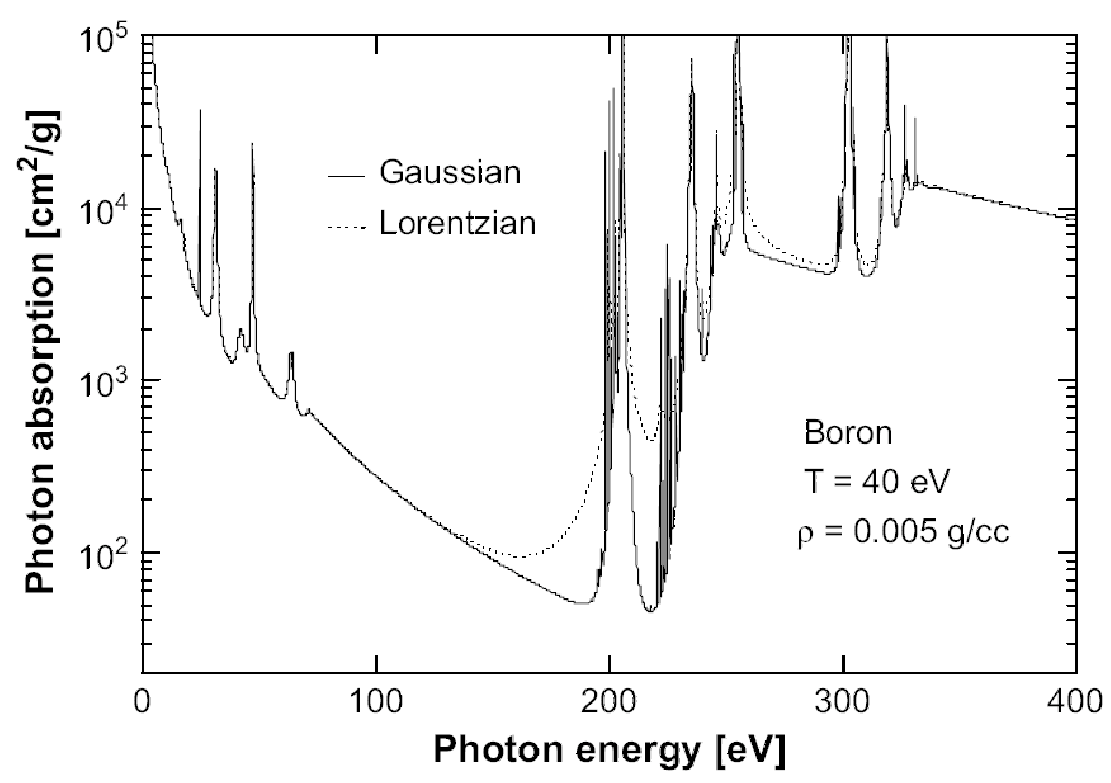

Figure 1. Opacity of a boron plasma (see [4] on p. 98)

the opacity $\sigma(\nu, T)$ is in general not known explicitly but tabulated. What is worse, the dependence of $\sigma(\nu, T)$ in either $\nu$ or $T$ is quite involved, and the function $\nu \mapsto \sigma(\nu, T)$ can be wildly oscillating, even for $T$ fixed, as can be seen on the graph given above.

We recognize in (4.1) the same type of difficulty that was handled in the two previous sections, since oscillations in the opacity $\sigma(\nu, T)$ are due to the dependence of that coefficient in the frequency $\nu$, while the streaming (or free transport) operator $\frac{1}{c} \partial_{t}+\omega \cdot \nabla_{x}$ acts on the variables $t$ and $x$ only.

Henceforth, we assume for simplicity that the temperature $T \equiv T(t, x)$ is given in the background medium which occupies the Euclidian space $\mathbf{R}^{3}$. We consider the following model problem:

$$
\left\{\begin{array}{l}
\frac{1}{c} \partial_{t} I_{\epsilon}+\omega \cdot \nabla_{x} I_{\epsilon}=\sigma_{\epsilon}(\nu, T) B_{\nu}(T)-\sigma_{\epsilon}(\nu, T) I_{\epsilon}, \\
\left.I_{\epsilon}\right|_{t=0}=I^{i n}(x, \omega, \nu),
\end{array}\right.
$$

posed for $(t, x, \omega, \nu) \in \mathbf{R}_{+}^{*} \times \mathbf{R}^{3} \times \mathbf{S}^{2} \times \mathbf{R}_{+}^{*}$. Here the oscillations of the opacity are recorded by the small parameter $\epsilon$ that is the typical "oscillation wavelength" in the variable $\nu$.

We henceforth assume that the given temperature profile $T$ is bounded away from 0 and $+\infty$, i.e. that $T \in[\theta, \Theta]$ for some constants $0<\theta<\Theta$, and that the family $\left(\sigma_{\epsilon}(\nu, T)\right)_{\epsilon>0}$ satisfies the uniform bound

$$
0<m \leq \sigma_{\epsilon}(\nu, T) \leq M, \quad \text { for each } \epsilon, \nu>0 \text { and } T \in[\theta, \Theta] .
$$

Furthermore, we assume that, for each $T>0$, the family $\sigma_{\epsilon}(\cdot, T)$ converges in the sense of Young measures to $\left(\mu_{\nu}^{T}\right)_{\nu>0}$ as $\epsilon \rightarrow 0^{+}$. By the method introduced in the previous section, we can formulate a theorem on the homogenized limit of the model problem (4.2) in the following manner. 
Theorem 4.1. In the limit as $\epsilon \rightarrow 0^{+}$, one has

$$
I_{\epsilon} \rightarrow I=\int_{0}^{+\infty} J d s \text { in } L^{\infty}\left(\mathbf{R}_{+} \times \mathbf{R}^{3} \times \mathbf{S}^{2} \times \mathbf{R}_{+}\right) \text {weak-* }
$$

where $J \equiv J(t, s, x, \omega, \nu)$ is the solution of

$$
\left\{\begin{array}{l}
\frac{1}{c} \partial_{t} J+\omega \cdot \nabla_{x} J-\partial_{s} J=\frac{d^{2} \tilde{\mu}_{\nu}^{T}}{d s^{2}} B_{\nu}(T), \\
\left.J\right|_{t=0}=-I^{i n}(x, \omega, \nu) \frac{d \tilde{\mu}_{\nu}^{T}}{d s}(s),
\end{array}\right.
$$

posed for $(t, s, x, \omega, \nu) \in \mathbf{R}_{+}^{*} \times \mathbf{R}_{+}^{*} \times \mathbf{R}^{3} \times \mathbf{S}^{2} \times \mathbf{R}_{+}^{*}$, where the notation $\tilde{\mu}_{\nu}^{T}$ denotes the Laplace transform of $\mu_{\nu}^{T}$.

The proof of this theorem is essentially the same as that of Proposition 3.1 - except for the source term in (4.2) - and we do not repeat it.

Observe that the homogenized problem (4.3) is a transport equation where the space variables are $x$ and $s$, and therefore defines a semigroup acting on the extended phase space $\mathbf{R}_{+} \times \mathbf{R}^{3} \times \mathbf{S}^{2} \times \mathbf{R}_{+}=\{(s, x, \omega, \nu)\}$, instead of the usual phase space $\mathbf{R}^{3} \times \mathbf{S}^{2} \times \mathbf{R}_{+}=\{(x, \omega, \nu)\}$ familiar in radiative transfer problems. More precisely, the solution of (4.3) is given in terms of the Duhamel formula for the transport semigroup in extended phase space defined by the left-hand side of that equation. This is at variance with the homogenized radiative transfer equations obtained in [5, 8] which are written in the usual phase space, but involve memory terms as in Tartar's example - and precisely for the same reason.

Notice that we have assumed that the initial data $I^{\text {in }}$ does not have fast oscillations in the $\nu$ variable - as is the case of $B_{\nu}$. In general, treating the case of an oscillating initial data $I_{\epsilon}^{i n}$ (in the $\nu$ variable, say) would require considering the joint Young measure of $I_{\epsilon}^{i n}$ and $\sigma_{\epsilon}$ - i.e. the Young measure of the couple $\left(I_{\epsilon}^{i n}, \sigma_{\epsilon}\right)$ viewed as a function of $\nu$ with values in $\mathbf{R}^{2}$. The complexity of the resulting model could be reduced in the case where the oscillations of $I_{\epsilon}^{i n}$ and $\sigma_{\epsilon}$ are independent so that the joint Young measure is the tensor product of the Young measure of $I_{\epsilon}^{i n}$ by that of $\sigma_{\epsilon}$.

A few words about the meaning of the additional variable $s$ appearing in the homogenized equation (4.3) are in order. The original equation (4.2) can be viewed as a balance equation for the number density of photons with frequency $\nu$ located at the position $x$ with direction $\omega$ at time $t$, that is $\frac{1}{c h \nu} I_{\epsilon}(t, x, \omega, \nu)$. The loss term $-\sigma_{\epsilon}(\nu, T) I_{\epsilon}(t, x, \omega, \nu)$ on the right-hand side of (4.2) models the absorption of photons by the matter as follows. Assuming for simplicity that $\sigma_{\epsilon} \equiv \sigma_{\epsilon}(\nu)$ is independent of temperature, the probability that a photon with frequency $\nu$ is not absorbed in the time interval $[0, t]$ is $e^{-t \sigma_{\epsilon}(\nu)}$. In the homogenized equation (4.3), the loss of photons due to absorption by the atoms of the surrounding matter is described by the term $-\partial_{s} J$ on the left-hand side. Any characteristic line of the streaming operator $\frac{1}{c} \partial_{t}+\omega \cdot \nabla_{x}-\partial_{s}$ being of the form $t \mapsto(x+c t \omega, s-t)$, the unknown quantity $\frac{1}{c h \nu} J(t, s, x, \omega, \nu)$ in (4.3) should be viewed as the number density at time $t$ of photons with frequency $\nu$ at the position $x$ in the direction $\omega$ which will be absorbed precisely at time $s+t$. In other words, in the homogenized model (4.3), the additional variable $s$ should be viewed as the "life expectancy" 
of photons, and their number density is disintegrated with respect to in probabilistic terms, conditioned relatively to - this new variable. The absorption of photons is described by characteristic lines of the streaming operator on the left-hand side of equation (4.3) leaving the phase space $s>0$, and not by prescribing the probability that a photon of frequency $\nu$ is absorbed in the infinitesimal interval of time $[t, t+d t]$.

\section{Conclusion}

We have explained how the notion of a "kinetic theory in extended phase space" introduced in [2] can be used in the homogenization problem for opacities in radiative transfer (Theorem 4.1), and how it avoids considering integro-differential equations whose solutions do not have the semigroup property, as in Tartar's elementary example.

The formalism presented here could be applied to various problems of the same nature. For instance, as mentioned above, opacities are strongly oscillating functions of the frequency variable, which seriously complicates the discretization of the radiative transfer equation. Usually, this is done by replacing the radiative intensity $I(\nu)$ with the vector $\left(I_{j}\right)_{1 \leq j \leq n}$, where

$$
I_{j} \simeq \int_{\nu_{j}}^{\nu_{j+1}} I(\nu) d \nu
$$

and where the frequency groups - i.e. the intervals $\left(\nu_{j}, \nu_{j+1}\right)$ - are chosen appropriately. Of course the main difficulty is to understand what to do with the absorption term

$$
\int_{\nu_{j}}^{\nu_{j+1}} \sigma(\nu) I(\nu) d \nu
$$

The projection of the radiative intensity on frequency groups as above is an instance of homogenization process, and one could hope that the considerations outlined in Theorem 4.1 might be helpful in this context.

Similar difficulties exist in the theory of neutron transport - with the neutron kinetic energy being the analogue of the frequency in radiative transfer. One could hope to apply the same method as above to this type of problem also; however, scattering processes are more important and should be taken into consideration, at variance with the discussion in the present paper. We hope to return to these questions in a forthcoming publication.

\section{REFERENCES}

[1] E. Bernard, E. Caglioti, F. Golse: Homogenization of the linear Boltzmann equation in a domain with a periodic distribution of holes; preprint arXiv:0906.4011. To appear in SIAM J. on Math. Anal..

[2] E. Caglioti, F. Golse: The Boltzmann-Grad limit of the periodic Lorentz gas in two space dimensions; C. R. Math. Acad. Sci. Paris 346 (2008), 477-482.

[3] F. Golse: On the periodic Lorentz gas in the Boltzmann-Grad scaling; Ann. Faculté des Sci. Toulouse 17 (2008), 735-749.

[4] C.A. Iglesias, V; Sonnad, B.G. Wilson, J.I. Castor: Frequency dependent electron collisional widths for opacity calculations; High Energy Dens. Phys. 5 (2009), 97104.

[5] C.D. Levermore, G.C. Pomraning, D.L. Sanzo, J. Wong: Linear transport theory in a random medium; J. Math. Phys. 27 (1986), 2526-2536. 
[6] J. Marklof, A. Strömbergsson: The Boltzmann-Grad limit of the periodic Lorentz gas; preprint arXiv:0801.0612.

[7] G.C. Pomraning: Linear kinetic theory and particle transport in stochastic mixtures; World Scientific, Singapore, 1991.

[8] R. Sentis: Homogénéisation des opacités en transfert radiatif; unpublished manuscript (1988).

[9] L. Tartar: Compensated compactness and applications to partial differential equations, Nonlinear analysis and mechanics: Heriot-Watt Symposium, Vol. IV, pp. 136212, Res. Notes in Math., 39, Pitman, Boston, Mass.-London, 1979.

[10] L. Tartar: An Introduction to Navier-Stokes Equation and Oceanography; Lecture Notes of the Unione Matematica Italiana 1, Springer, Berlin, Heidelberg, New-York, 2006.

[11] D. Vanderhaegen: Radiative transfer in statistically heterogeneous mixtures; J. Quant Spectrosc. Radiative Transf. 36 (1986), 557-561.

[12] D. Vanderhaegen: Impact of a mixing structure on radiative transfer in random media; J. Quant Spectrosc. Radiative Transf. 39 (1988), 333-337.

(E. B.) Ecole polytechnique, Centre de mathématiques L. Schwartz, F91128 Palaiseau cedex, \& Université P.-et-M. Curie, Laboratoire J.-L. Lions, BP 187, 75252 PARIS CEDEX 05

E-mail address: etienne.bernard@math.polytechnique.fr

(F. G.) Ecole polytechnique, Centre de mathématiques L. Schwartz, F91128 Palaiseau cedex, \& Université P.-et-M. Curie, Laboratoire J.-L. Lions, BP 187, F75252 PARIS CEDEX 05

E-mail address: francois.golse@math.polytechnique.fr

(F. S.) Dipartimento di Matematica F. Casorati, Università degli Studi di Pavia, Via Ferrata 1, 27100 Pavia, Italia

E-mail address: francesco.salvarani@unipv.it 